\title{
Actuarial Model of the Implicit Pension Debt of China with the Stochastic Interest Rate
}

\author{
N.N. Jia, J.B. Yang \& H. Yang \\ College of Science, Harbin Engineering University, Harbin, 150001, China \\ ajianiannian@hrbeu.edu.cn, b793967214@qq.com, c498989451@qq.com
}

Keywords: stochastic interest rate, implicit pension debt, Monte Carlo simulation

\begin{abstract}
In the global context of population aging, the problem of the implicit pension debt is gradually dominant in China. Firstly, based on the actuarial model of the implicit pension debt with the fixed interest rate, we establish the actuarial model of the implicit pension debt with the stochastic interest rate. Secondly, with the model, the expression of expectation is derived. Finally, Monte Carlo simulation is performed. The result provides a theoretical basis to estimate the exact size of implicit pension debt for Chinese government.
\end{abstract}

\section{Introduction}

Within actuarial theory, the research of implicit pension debt is gaining greater importance [1]. Zhen Li studied IPD (Implicit Pension Debt) of China by using the method of rough estimation in early [2]. Qiwen Zhang (2012) calculated the new rural social pension insurance of some north province in our country [3]. Burkhard Heer and Andreas Irmen (2014) discovered that the results of implicit pension debt scale calculation have difference because of the economic growth in different ways [4].

While, the realistic interest rate is random now, so the actuarial model with the fixed interest rate has some limitations in actual operation process. This paper builds the actuarial model of the implicit pension debt with the stochastic interest rate.

\section{Model}

\section{The actuarial model of the implicit pension debt with the fixed interest rate}

According to Chinese National document [2005] No.38, the insured population is divided into four parts.

"The old people" refer to the insured population who had retired before Chinese National document [1997] No.26 implemented [5]. The implicit pension debt of "the old people" $\left(I P D_{1}\right)$ can be expressed as

$$
I P D_{1}=\sum_{x=r+t-n}^{\omega-1} L O_{x, t} \cdot(P V F B)_{x, t}^{(1)}
$$

where, $(P V F B)_{x, t}^{(1)}=\sum_{k=1}^{\omega-x} B_{0}\left(1+\phi \cdot g_{y}\right)^{k} v^{k}{ }_{k} p_{x}$ is the actuarial present value that "the old people" in the age of $x$ pay prospective pension at time $t ; B_{0}$ is the amount of annual pension payment of "the old people" $; \phi$ is adjustment coefficient of the growth rate of income; $g_{y}$ is growth rate of income on average; $L O_{x, t}$ is the amount of "the old people" at time $t ; v$ is discount rate; ${ }_{k} p_{x}$ is the probability that people in the age of $x$ live longer than $x+k$ years[6]; $\omega$ is the age of limitation; $r$ is the age of retire; $n$ is the year that new system started.

"The people in transition" refer to the insured population who had worked before Chinese National document [1997] No.26 implemented, and retired before Chinese National document [2005] No.38 implemented [5]. The implicit pension debt of "the people in transition" includes two parts, transitional pension, and the basic pension. Accordingly, the IPD of "the people in transition" is

$$
I P D_{2}=I P D_{21}+I P D_{22}
$$




$$
\begin{aligned}
& =\sum_{x=r+t-2005}^{r+t-n-1} L_{x, t} \cdot(P V F B)_{x, t}^{(2)} \\
& +\sum_{x=r+t-2005}^{r+t-n-1} L O M_{x, t} \cdot\left[(P V F B)_{x, t}^{(3)} \cdot \frac{x-a-(t-n)}{\bar{H}}\right]
\end{aligned}
$$

where, $(P V F B)_{x, t}^{(2)}=\varepsilon \cdot \beta \cdot \bar{W} \cdot T I_{x} \sum_{k=0}^{\omega-x-1} v^{k}{ }_{k} p_{x}$ is the actuarial present value that "the people in transition" in the age of $x$ pay prospective transitional pension at time $t ; L_{X O M_{x, t}}$ is the amount of "the people in transition" at time $t ; \varepsilon$ is the proportion of the planned payment of transitional pension; $\beta$ is the index of payment on average; $\bar{W}$ is one's last-year social average salary that can be got at the time of retire; $T I_{x}$ is the payment or the period which can be seen as the payment year when one enter the plan of individual account; $(P V F B)_{x, t}^{(3)}=\lambda \cdot \bar{W} \cdot \sum_{k=0}^{\omega-x-1}\left(1+\phi \cdot g_{y}\right)^{k} v^{k}{ }_{k} p_{x}$ is the actuarial present value that "the people in transition" in the age of $x$ pay prospective basic pension at time $t ; a$ is the age of the insured population; $\bar{H}$ is the time at work on average; $\lambda$ is the proportion of the planned payment of pension. "The new retired people" refer to the insured population who had worked before Chinese National document [1997] No.26 implemented, and retired before time $t$ [5]. The IPD of "the new retired people" $\left(I P D_{3}\right)$ can be given by

$$
I P D_{3}=I P D_{31}+I P D_{32}
$$

$$
\begin{aligned}
& =\sum_{x=r}^{r+t-2006} L_{N R M_{x, t}} \cdot(P V F B)_{x, t}^{(4)} \\
& +\sum_{x=r}^{r+t-2006} L N R M_{x, t} \cdot\left[(P V F B)_{x, t}^{(5)} \cdot \frac{x-a-(t-n)}{\bar{H}}\right]
\end{aligned}
$$

where, $(P V F B)_{x, t}^{(4)}=\varepsilon \cdot \beta \cdot \bar{W} \cdot T I_{x} \sum_{k=0}^{\omega-x-1} v^{k}{ }_{k} p_{x}$ is the actuarial present value that "the new retired people" in the age of $x$ pay prospective transitional pension at time $t ; L N R M_{x, t}$ is the amount of "the new retired people" at time $t ;(P V F B)_{x, t}^{(5)}=\frac{\overline{W_{0}}+\overline{W_{i}}}{2} \cdot T I \cdot 1 \% \cdot \sum_{k=0}^{\omega-x-1}\left(1+\phi \cdot g_{y}\right)^{k} v^{k}{ }_{k} p_{x}$ is the actuarial present value that "the new retired people" in the age of $x$ pay prospective basic pension at time $t ; \bar{W}_{0}$ is one's last-year average salary that can be got at work; $\bar{W}_{i}$ is the indexing payment salary on average; TI is the payment or the period which can be seen as the payment year when one retire.

"The new in-service people" refer to the insured population who had worked before Chinese National document [1997] No.26 implemented, and retired after time $t$ [5]. The IPD of "the new in-service people" $\left(\mathrm{IPD}_{4}\right)$ is

$$
\begin{aligned}
& I P D_{4}=I P D_{41}+I P D_{42} \\
= & \sum_{x=a+t-n}^{r-1} L N M_{x, t} \cdot(P V F B)_{x, t}^{(6)} \\
+ & \sum_{x=a+t-n}^{r-1} L N M_{x, t} \cdot\left[(P V F B)_{x, t}^{(7)} \cdot \frac{x-a-(t-n)}{\bar{H}}\right]
\end{aligned}
$$

where, $(P V F B)_{x, t}^{(6)}=\varepsilon \cdot \beta \cdot T I_{x} \cdot W_{t-1} \cdot\left(1+g_{y}\right)^{r-x-1} \cdot{ }_{r-x} p_{x} \cdot v^{r-x} \cdot \sum_{k=0}^{\omega-1-r} v^{k}{ }_{k} p_{r}$ is the actuarial present value that "the new in-service people" in the age of $x$ pay prospective transitional pension at average salary at time $t-1 ;(P V F B)_{x, t}^{(7)}=\frac{\bar{W}_{0}+\bar{W}_{i}}{2} \cdot T I \cdot 1 \% \cdot{ }_{r-x} p_{x} \cdot v^{r-x} \cdot \sum_{k=0}^{\omega-1-r}\left(1+\phi \cdot g_{y}\right)^{k} v^{k}{ }_{k} p_{r}$, is the actuarial present value that "the new in-service people" in the age of $x$ pay prospective basic pension at time $t$. Thus, we can get the actuarial model of IPD $I P D=I P D_{1}+I P D_{2}+I P D_{3}+I P D_{4}$

$$
\begin{aligned}
& =\sum_{x=r+t-n}^{\omega-1} L O_{x, t} \cdot \sum_{k=1}^{\omega-x} B_{0}\left(1+\phi \cdot g_{y}\right)^{k} v^{k}{ }_{k} p_{x}+\sum_{x=r+t-2005}^{r+t-n-1} L O M_{x, t} \cdot\left[\varepsilon \cdot \beta \cdot \bar{W} \cdot T I_{x} \sum_{k=0}^{\omega-x-1} v^{k}{ }_{k} p_{x}\right. \\
& \left.+\lambda \cdot \bar{W} \cdot \sum_{k=0}^{\omega-x-1}\left(1+\phi \cdot g_{y}\right)^{k} v^{k}{ }_{k} p_{x} \cdot \frac{x-a-(t-n)}{\bar{H}}\right]+\sum_{x=r}^{r+t-2006} L N R M_{x, t}
\end{aligned}
$$




$$
\begin{aligned}
& \cdot\left[\varepsilon \cdot \beta \cdot \bar{W} \cdot T I_{x} \sum_{k=0}^{\omega-x-1} v^{k}{ }_{k} p_{x}+\frac{\overline{W_{0}}+\bar{W}_{i}}{2} \cdot T I \cdot 1 \% \cdot \sum_{k=0}^{\omega-x-1}\left(1+\phi \cdot g_{y}\right)^{k} v^{k}{ }_{k} p_{x} \cdot \frac{x-a-(t-n)}{\bar{H}}\right](5) \\
& +\sum_{x=a+t-n}^{r-1} L_{N M_{x, t}} \cdot\left[\varepsilon \cdot \beta \cdot T I_{x} \cdot W_{t-1} \cdot\left(1+g_{y}\right)^{r-x-1} \cdot{ }_{r-x} p_{x} \cdot v^{r-x} \cdot \sum_{k=0}^{\omega-1-r} v^{k}{ }_{k} p_{r}\right. \\
& \left.+\frac{\overline{W_{0}}+\overline{W_{i}}}{2} \cdot T I \cdot 1 \% \cdot{ }_{r-x} p_{x} \cdot v^{r-x} \cdot \sum_{k=0}^{\omega-1-r}\left(1+\phi \cdot g_{y}\right)^{k} v^{k}{ }_{k} p_{r} \cdot \frac{x-a-(t-n)}{\bar{H}}\right]
\end{aligned}
$$

\section{The actuarial model of the implicit pension debt with the stochastic interest rate}

\section{Model foundation}

Lemma 1: Let $W(t)$ is a standard Wiener process, $a \geq 0$ is constant, then

$$
E_{W}\left(e^{a W(t)}\right)=e^{\frac{1}{2} a^{2} t}
$$

Assuming that the accumulation function of interest force is $\quad R(t)=\delta t+\beta W(t)(0 \leq t \leq \infty)$ where, $W(t)$ is the Wiener Process, $\beta$ is constant parameter, $\delta$ is constant interest force. Then we can derive the expectation of IPD according to combine formula (5) with lemma 1

$$
\begin{aligned}
& E(I P D)=\sum_{x=r+t-n}^{\omega-1} L O_{x, t} \cdot \sum_{k=1}^{\omega-x} B_{0}\left(1+\phi \cdot g_{y}\right)^{k} e^{-\delta k-\frac{1}{2} \beta^{2} k} p_{x}+\sum_{x=r+t-2005}^{r+t-n-1} L O M_{x, t} \cdot\left[\varepsilon \cdot \beta \cdot \bar{W} \cdot T I_{x} \sum_{k=0}^{\omega-x-1} e^{-\delta k-\frac{1}{2} \beta^{2} k}{ }_{k} p_{x}\right. \\
& \left.+\lambda \cdot \bar{W} \cdot \sum_{k=0}^{\omega-x-1}\left(1+\phi \cdot g_{y}\right)^{k} e^{-\delta k-\frac{1}{2} \beta^{2} k}{ }_{k} p_{x} \cdot \frac{x-a-(t-n)}{\bar{H}}\right]+\sum_{x=r}^{r+t-2006} L N R M_{x, t} \cdot\left[\varepsilon \cdot \beta \cdot \bar{W} \cdot T I_{x} \sum_{k=0}^{\omega-x-1} e^{-\delta k-\frac{1}{2} \beta^{2} k}{ }_{k} p_{x}+\frac{\overline{W_{0}}+\overline{W_{i}}}{2} \cdot T I\right. \\
& \left.\cdot 1 \% \cdot \sum_{k=0}^{\omega-x-1}\left(1+\phi \cdot g_{y}\right)^{k} e^{-\delta k-\frac{1}{2} \beta^{2} k}{ }_{k} p_{x} \cdot \frac{x-a-(t-n)}{\bar{H}}\right]+\sum_{x=a+t-n}^{r-1} L N M_{x, t} \cdot\left[\varepsilon \cdot \beta \cdot T I_{x} \cdot W_{t-1}\right. \\
& \cdot\left(1+g_{y}\right)^{r-x-1} \cdot{ }_{r-x} p_{x} \cdot e^{-\delta(r-x)-\frac{1}{2} \beta^{2}(r-x)} \cdot \sum_{k=0}^{\omega-1-r} e^{-\delta k-\frac{1}{2} \beta^{2} k}{ }_{k} p_{r}+\frac{W_{0}+\overline{W_{i}}}{2} \\
& \left.\cdot T I \cdot 1 \% \cdot{ }_{r-x} p_{x} \cdot e^{-\delta(r-x)-\frac{1}{2} \beta^{2}(r-x)} \cdot \sum_{k=0}^{\omega-1-r}\left(1+\phi \cdot g_{y}\right)^{k} e^{-\delta k-\frac{1}{2} \beta^{2} k}{ }_{k} p_{r} \cdot \frac{x-a-(t-n)}{\bar{H}}\right]
\end{aligned}
$$

\section{The Monte Carlo simulation of the implicit pension debt with the stochastic interest rate}

This paper selects the beginning of January 1, 2013 as the benchmark moment, studies one group consists of $c$, divides the group into $m$ teams. People in same team have the same age $x_{i}$, the sum of pension when people retire $B_{i}$ and the time when they begin to get pension. We first assume as follows:

$c_{i}$ : The number of people in team $i ; n_{i}$ : The time that team $i$ get the pension at first from National document [1997] No.26 issued; $r$ : If the people is male, $r=60$.If the people is female, $r=55$; TI : If people is male, $T I=40$.If people is female, $T I=35 ; T I_{x}=a-32 ; g_{y}=11 \%, \phi=0.8, \varepsilon=1.2 \%, \beta=100 \%$, $\overline{W_{0}}=\overline{W_{i}}=\bar{W}=46769, \lambda=20 \%$.In this section, we study a group with 10000 members to apply the results that have deduced. We divide them into 10 teams. The constituents of the group are shown in Table 1. 
Table 1: The constituents of the group

\begin{tabular}{|l|l|l|l|l|l|}
\hline$i$ & $x_{i}$ & $n_{i}$ & $B_{i}$ & $c_{i}$ & Sex \\
\hline 1 & 60 & 0 & 14700 & 750 & Male \\
\hline 2 & 55 & 0 & 14700 & 1050 & Female \\
\hline 3 & 65 & 0 & 16538 & 750 & Male \\
\hline 4 & 70 & 0 & 18375 & 650 & Male \\
\hline 5 & 60 & 0 & 16800 & 950 & Female \\
\hline 6 & 35 & 25 & 5513 & 1500 & Male \\
\hline 7 & 35 & 20 & 6300 & 1350 & Female \\
\hline 8 & 50 & 10 & 11025 & 1100 & Male \\
\hline 9 & 40 & 15 & 8400 & 1000 & Female \\
\hline 10 & 45 & 10 & 10500 & 900 & Female \\
\hline
\end{tabular}

It is difficult to obtain the precise distribution as the expression of IPD because of its complex. In order to solve this problem, this paper adopts Monte Carlo simulation to seek the empirical distribution of IPD. According to 10000 analogue of IPD Monte Carlo simulation produced, we can obtain the diagram of empirical distribution function and the diagram of density function of IPD under building models with the stochastic interest rate. The results are as follows:

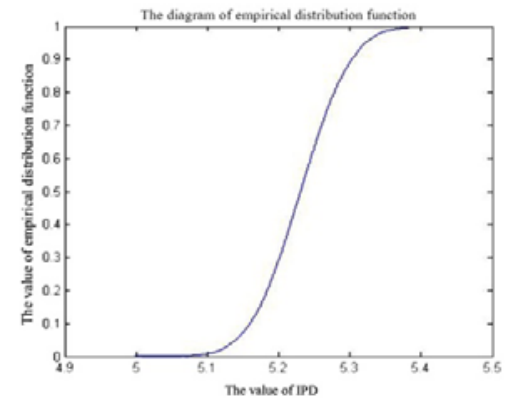

(a)

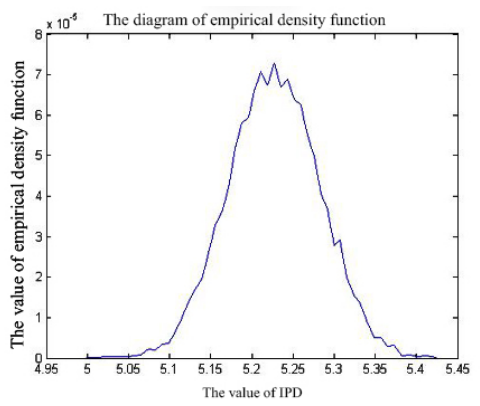

(b)

Fig.1: Under the standard Wiener process, (a) is the diagram of empirical distribution function and (b) is the diagram of density function of IPD

According to the simulation value of IPD, we can conclude that the trends of IPD with the standard Wiener process. It shows that the model we choose is reasonable. Thus, Chinese government should choose the appropriate stochastic interest rate combining with society, market, policy of China, and international environment. In such cases, determining the exact size of the implicit pension debt is particularly important.

\section{Conclusion}

This paper studies implicit pension debt of China. Firstly, the paper builds actuarial model of implicit pension debt with the fixed interest rate model. Secondly, this paper uses standard Wiener process to build the accumulation function of interest force, deduces the expectation of the stochastic interest rate. Finally, the paper applies Monte Carlo to simulate the model with the stochastic interest, obtain the diagram of empirical distribution function and the diagram of empirical density function of IPD. The results provide more realistic theory basis for Chinese finical institutions.

\section{References}

[1] R. Na:The understanding about the implicit pension debt of China. Journal of base (2006), p. 93.

[2] Y.Y. Qi and M. Tao:Actuarial analysis about the implicit pension debt of the social pension system in current country [J].Journal of Population (2011), p. 68-74.

[3] Q.W. Zhang, H.F. Li and L.N. Gao:Study on improving development strategies of new rural social pension insurance system in Heilongjiang Province[J]. Journal of Northeast Agricultural University (English Edition), 19 (2012), p. 83-88. 
[4] B. Heer and A. Irmen: Population, pensions, and endogenous economic growth. Journal of Economic Dynamics and Control (2014), p. 50-72.

[5] Y.X. Wang, H. Wang and J. Xiao:Forecast on population distribution of Shanghai pension system based on the gray GM $(1,1)$ model. System Engineering -Theory and Practice, 30 (2014), p. 2244-2248, .

[6] X.J. Wang: Actuarial science of life insurance (Chinese People's University Publications, Beijing 2005). 